\title{
Filigrane
}

Écoutes psychanalytiques

\section{Cadre interne, transfert et contre-transfert}

\section{Jean-François Chiantaretto}

\section{Volume 27, numéro 2, 2018}

La consultation psychanalytique aujourd'hui, entre héritages et remaniements

URI : https://id.erudit.org/iderudit/1055752ar

DOI : https://doi.org/10.7202/1055752ar

Aller au sommaire du numéro

\section{Éditeur(s)}

Revue Santé mentale au Québec

ISSN

1192-1412 (imprimé)

1911-4656 (numérique)

Découvrir la revue

Citer cet article

Chiantaretto, J.-F. (2018). Cadre interne, transfert et contre-transfert. Filigrane, 27(2), 91-100. https://doi.org/10.7202/1055752ar

\section{Résumé de l'article}

Les modalités spécifiques du transfert et du contre-transfert dans le champ de la psychopathologie des limites seront ici abordées sous l'angle de l'interlocution interne de l'analyste en séance. L'hypothèse d'une « puissance instituante du transfert de l'analyste " permettra d'aborder les enjeux liés à l'idée d'une offre de l'analyste conditionnant la demande de l'analysant. À ce titre, chaque cure convoque une scène originaire : le rapport de l'analyste aux origines de la psychanalyse, du commencement freudien au re-commencement ferenczien.
Ce document est protégé par la loi sur le droit d'auteur. L'utilisation des services d'Érudit (y compris la reproduction) est assujettie à sa politique d'utilisation que vous pouvez consulter en ligne.

https://apropos.erudit.org/fr/usagers/politique-dutilisation/ 


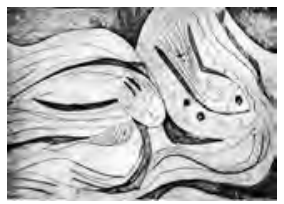

\title{
Cadre interne, transfert et contre-transfert
}

\author{
Jean-François Chiantaretto
}

\begin{abstract}
Résumé: Les modalités spécifiques du transfert et du contre-transfert dans le champ de la psychopathologie des limites seront ici abordées sous l'angle de l'interlocution interne de l'analyste en séance. L'hypothèse d'une "puissance instituante du transfert de l'analyste» permettra d'aborder les enjeux liés à l'idée d'une offre de l'analyste conditionnant la demande de l'analysant. À ce titre, chaque cure convoque une scène originaire: le rapport de l'analyste aux origines de la psychanalyse, du commencement freudien au re-commencement ferenczien.
\end{abstract}

Mots clés: meurtre psychique; psychopathologie des limites; scène originaire; transfert de l'analyste; travail de la mélancolie.

\begin{abstract}
In this paper, the specific modalities of transference and countertransference in the field of the psychopathology of the limits will be approached from the angle of the internal interlocution of the analyst in session. The hypothesis of an "instituting power of the transference of the analyst" will make it possible to address the issues related to the idea of an analyst's offer conditioning the analysand's request. As such, each cure brings together an original scene: the analyst's relationship to the origins of psychoanalysis, from the Freudian beginning to the Ferenczian re-beginning.
\end{abstract}

Keywords: psychic murder; psychopathology of limits; original scene; transference of the analyst; work of melancholy.

e cadre interne sera ici envisagé sous l'angle de l'interlocution interne de l'analyste en séance, dans la perspective que je propose depuis 2002 (Chiantaretto, 2006, 2011). La question du transfert de l'analyste avec les fonctionnements limites sera au cour de mon propos; à partir d'une pratique libérale, mais avec l'espoir de pouvoir croiser quelques-uns des enjeux de la consultation psychanalytique au sein de centres proposant un traitement psychanalytique.

\section{La puissance instituante du transfert de l'analyste}

Il y aurait à distinguer rencontre et premier entretien, distinction qui a amené Donnet et Baldacci à envisager la dimension analytique de la rencontre comme étant toujours dans l'après-coup. Cette distinction entre 
rencontre et premier entretien prend en outre une consistance spécifique dans un centre de consultations, avec la différenciation ou non entre l'analyste qui reçoit dans un premier temps la demande et l'analyste qui assure le traitement. Dans un centre de consultations différenciant analyste «consultant» et analyste «traitant», la différenciation a été définitivement théorisée par Donnet puis Baldacci, avec l'idée de «double cadre», soit «le cadre du cadre défini par le consultant en fonction des règles de l'institution» (Baldacci, 2010, p. 65). Mais sous une forme certes particulière, la question posée en arrière-fond reste bien celle des conditions requises, dans toute pratique psychanalytique, en ou hors institution, pour mettre en ouvre l'écart aussi bien entre le cadre et l'analyste qu'entre l'analyste comme personne et l'analyste interprète - écarts spécialement attaqués avec les fonctionnements limites.

Dans cette perspective, il y aurait à mettre en rapport ce qui se joue côté patient et côté analyste. Côté patient: avec les fonctionnements limites, la nécessité d'un tiers à attaquer en personne chez l'analyste, pour éprouver - dans les deux sens du terme - la distinction entre l'analyste comme personne et l'analyste destinataire du transfert; côté analyste: la fonction tierce de la théorie dans le transfert et/ou le contre-transfert. Ou, pour le dire autrement: côté patient, les conditions de possibilité de l'expérience d'un décalage entre l'adresse transférentielle dans le discours intérieur et l'adresse de sa parole, expérience qui suppose le plus souvent le face à face, dans le registre limite; côté analyste, les conditions de possibilité de l'expérience d'un décalage entre ses affects contre-transférentiels et son propre transfert, en tant qu'il ouvre la voie du transfert du patient et ouvre la voix de l'interprétation dans le transfert. Cette expérience intérieure de l'analyste, attaquée par le patient limite et précisément en ce qu'elle est attaquée par lui, conditionne la possibilité de l'expérience analysante de l'intériorité - de l'autre, de soi. Cette expérience intérieure de l'analyste: un registre qui passe par les mots, par l'écoute de son écoute et de ce qui la déborde, à la fois en lui et au-delà de lui. Il s'agit bien de ce que je nomme l'interlocution interne de l'analyste, telle qu'elle met en ouvre son altérité interne et appelle celle de l'analysant, lesquelles tendent précisément à être confondues dans une même attaque par les patients limites.

J'esquisserais l'hypothèse d'une puissance instituante du transfert de l'analyste, faisant intrinsèquement partie de la donne analytique, indépendamment de tout dispositif institutionnel - une puissance à la fois mise en question et prenant sa visibilité maximale avec les fonctionnements limites. 
Une telle hypothèse se situerait dans la ligne des travaux de Green et Neyraut, qui chacun à leur manière théorisent la précession du contre-transfert sur le transfert, de l'offre contre-transférentielle sur la demande transférentielle - Neyraut allant même jusqu'à affirmer que non seulement le contre-transfert n'est pas une réponse, mais qu'il constitue une "demande» (Neyraut, 1974, p. 25).

Il ne s'agit pas seulement de considérer que transfert du patient et contre-transfert de l'analyste forment une unité, ni même seulement de donner toute sa place à l'affect dans le contre-transfert, en prenant acte avec Neyraut que les affects contre-transférentiels sont à l'origine de l'acte interprétatif (Neyraut, 1974, p. 53). Le contre-transfert ne se limite pas aux impacts affectifs du transfert du patient, mais implique l'ensemble du fonctionnement psychique de l'analyste en situation. Il y aurait à prendre la mesure d'une évolution depuis Freud de l'idée du contre-transfert comme butée, une évolution en bonne partie liée à la psychopathologie des limites et faisant du contre-transfert un instrument privilégié, un élément fondamental du cadre, le point d'appui de toute interprétation. L'analyste peut aujourd'hui se considérer comme ayant à «répondre du transfert et en assumer la responsabilité» (Guyomard, 2011, p. 14), selon l'excellente formule de Guyomard dans sa lecture de Lacan, chez qui il met en relief l'idée de l'analyse comme rencontre du désir du patient et du désir de l'analyste. Et Guyomard insiste pour souligner que chez Lacan l'idée que le désir de l'analyste précède le transfert ne fait pas disparaître le terme de contre-transfert.

Autrement dit, la question, même chez Lacan, n'est pas de récuser le contre-transfert - ni le terme, ni l'idée -, mais l'insuffisance du terme pour rendre compte de la dimension transférentielle de l'implication de l'analyste et de sa responsabilité vis-à-vis du transfert du patient. Il y a bien lieu de ne pas réduire le contre-transfert à une réaction ou une réponse, et de le considérer plutôt comme un appel à un positionnement interne permettant de soutenir une double responsabilité: quant à son implication dans et par le transfert du patient d'une part, quant à l'auto-observation après coup de cette implication d'autre part. Et c'est ici que le terme «contre-transfert» ne suffirait plus, au sens où cette double responsabilité est creusée par le travail lié à la confrontation de l'analyste à son inanalysé: une responsabilité au travail, à conquérir comme source d'animation et point de fuite de l'élaboration du contre-transfert.

Il s'agit pour l'analyste, selon Fédida, d'imaginer ce que ressent le patient comme semblable (Fédida, 2002, p. 92), mais j'ajouterais qu'il s'agit 
aussi de continuer à imaginer son propre analyste, avec chaque patient différemment, ce qui suppose de parvenir suffisamment à s'auto-observer et s'auto-questionner, dans l'actualité de sa position d'analyste et dans l'actualisation de son passé analysant. Autrement dit, le travail élaboratif de l'analyste supposerait d'imaginer son propre analyste à travers l'observation de ses résistances face au patient, résistances qui désigneraient et dessineraient un inanalysé en résonance avec l'inanalysé de son (ses) analyste(s).

La confrontation de l'analyste avec le destin en lui de l'inanalysé de son propre analyste est imposée par les patients limites. Car ils ont besoin, pour abandonner leur invulnérabilité et parvenir à souffrir (Winnicott, 1967, p. 326), et donc, pour pouvoir s'identifier à l'analyste, que celui-ci fasse l'expérience de sa vulnérabilité et de son refoulement, tout en maintenant ce que Winnicott appelle une "attitude professionnelle» (Winnicott, 1960). Je reformulerais l'attitude professionnelle comme une disposition chez l'analyste à l'auto-observation de l'énigme touchée en lui par le patient, de ses liens identificatoires à son propre analyste et à son inanalysé. Cette auto-observation passe par un discours intérieur, une interlocution interne garante de «l'étranger intime», du «site de l'étranger» dont parle Fédida (1995), qui consiste dans le maintien de l'écart entre le sujet de l'écoute et le sujet destinataire de la parole - le sujet supposé savoir?

L'altérité interne maintenue à l'œuvre chez l'analyste est la condition du maintien d'une dissymétrie entre l'analyste et le patient - dissymétrie que le patient limite, en toute incestualité, et attaque en utilisant les limites de l'analyste comme la preuve de la symétrie des places. Le patient limite veut légitimer la confusion entre l'analyste interprète et l'analyste tuable, cible de la haine de transfert et, à ce titre, condition de possibilité d'une visée susceptible de faire du meurtre le motif d'une absence. Comment ne pas laisser tuer la possibilité symbolique du meurtre?, telle serait la question pour l'analyste face à un patient limite. Lorsque Baldacci, à la suite de Donnet, parle d'une «fonction tiercéisante du processus», lorsqu'il traduit le désir de l'analyste selon Lacan en "transfert sur la psychanalyse» (Baldacci, 2016), je dirais quant à moi que cela renvoie à un transfert originel. Un transfert à l'origine de la psychanalyse, support d'une scène originaire pour l'analyste et constitutif de sa position même: l'entre-deux matérialisé par la relation analytique impossible de Freud et Ferenczi. 


\section{Après Freud et Ferenczi : donner un lieu au meurtre pour qu'il n'ait pas lieu}

L'interlocution interne de l'analyste, telle que je la définis, correspond pour partie à la réitération, par et pour chaque analyste, du dialogue créateur au commencement de la psychanalyse: Freud anticipant ses lecteurs, sollicités comme témoins et interlocuteurs d'un penser en cours. Mais au re-commencement sera le meurtre. Au re-commencement de la psychanalyse dans la relation analytique impossible de Freud et Ferenczi, il y a une résistance meurtrière à la psychanalyse. La possibilité du meurtre trouve un mi-accomplissement: un meurtre psychique - un meurtre d'âme? - est commis à deux; Freud et Ferenczi, ne visant ni seulement Freud ni seulement Ferenczi, mais plutôt la relation analytique Freud/Ferenczi. Elle est tout à la fois impossible, condamnée à rester une relation (passionnelle), et elle vient montrer la puissance, créatrice et possiblement auto-destructrice, de la polarité à l'œuvre dans la psychanalyse, de la dualité au cœur même du processus de sa création. Cette dualité créatrice, d'abord individuelle avec Freud, se prolonge à deux, dans l'alliance transférentielle, par nature inanalysable, du commencement freudien et du re-commencement ferenczien (Chiantaretto, 2016).

Freud est un analyste inanalysable, pour lequel la place de l'analyste est occupée et barrée par l'invention de la psychanalyse. Mais Ferenczi est lui-même un analysant inanalysable. Là où Freud efface la possibilité d'un analyste pour lui en inventant la psychanalyse, Ferenczi ne peut que tenter désespérément de réinventer la psychanalyse pour remédier à l'impossibilité pour Freud d'être son analyste, lui qui ne peut avoir d'analyste! En d'autres termes, Ferenczi ne peut que tenter - en y échouant - de combler la place vide de l'analyste de son analyste par la création théorique, à laquelle il demande l'impossible: se faire l'analyste de Freud pour faire de Freud son analyste. L'échec est dramatique: il n'y aura personne pour accueillir la haine meurtrière de l'enfant-thérapeute mal accueilli. Elle sera mise en demeure de rester hors sujet, entre l'un et l'autre, avec pour seule affectation chez Ferenczi de transformer son impossible affectation en une source de pensée créatrice - au prix que l'on sait.

Le dialogue interne de Freud et son relais par le dialogue avec Ferenczi vient désigner une place virtuelle, celle de l'analyste de Freud. Cette place, qui pour Ferenczi prend statut de place vide, doit être investie comme vacante - et non pas vide - par les analystes. Ils se doivent de résister à l'irréductible tentation de l'occuper, liée à leur position de successeurs de Freud et d'héritiers du dialogue Freud/Ferenczi. L'enjeu est de poursuivre le dialogue 
interne de Freud, à la suite de Ferenczi, mais autrement. Il leur faut pour cela accepter de permettre à leur dialogue impossible de se poursuivre et de rester créateur en eux. Cela implique d'accepter la responsabilité d'œuvrer à partir du destin en eux de l'inanalysé de leurs analystes, en tant qu'il ouvre l'accès à leur propre inanalysé - dans le questionnement sur leur fonctionnement psychique, tel qu'il est amené par chaque patient, entre le su et l'insu, dans la tension inconfortable entre le déjà pensé et le non encore pensable. En d'autres termes, il s'agit pour chacun de consentir au meurtre de l'analyste de l'analyste, un meurtre symbolique, symboligène et supposé partageable au sein de la communauté analytique... La communauté analytique, c'està-dire un idéal, un au-delà exigeant de s'incarner dans chaque cure, au-delà donc des logiques d'appartenance.

\section{Recommencer le meurtre, re-commencer à penser le meurtre: un échec clinique}

Je voudrais ré-évoquer autrement la situation clinique présentée dans le texte signalé plus haut, autour de «L'enfant mal accueilli et sa pulsion de mort» (Chiantaretto, 2016). J'insisterai ici sur l'effet d'après-coup lié précisément au fait d'avoir déjà présenté cette situation, la présentation ayant eu un effet élaboratif.

La patiente, clinicienne en début de formation analytique, m'a été adressée par une analyste ayant été ma patiente, avec qui elle a fait une longue analyse. Le second entretien préliminaire a été particulièrement marquant, annonçant la suite, en rupture avec un premier entretien où une hystérie plutôt bien tempérée semblait occuper le devant de la scène. Au bout de quelques minutes, la patiente littéralement s'écroule, se laissant glisser du fauteuil par terre, avec des sanglots et des spasmes plus ou moins retenus, sans parler. Elle se relèvera seulement au moment où je lui signifierai la fin, en se reprenant assez facilement.

J'interpréterai après coup mon éprouvé, de l'ordre de la sidération, comme la lutte en moi de deux types de scène interprétative: une scène très sexualisée, plutôt à dominante phallique, appelant mon regard; une scène sans personne, où gisait sur le sol une marionnette dont les fils n'étaient plus tenus. Et la tension entre ces deux scènes m'apparaîtra dans le travail d'écriture, comme le lieu même de mon offre au transfert, désignant plus précisément encore le cœur transférentiel de mon contre-transfert - cœur transférentiel qui aura animé mon contre-transfert tout autant qu'il l'aura creusé, en activant l'effacement d'affects contre-transférentiels trop envahissants. 
Deux ans après, la catastrophe annoncée a eu lieu et plusieurs épisodes suicidaires graves se sont succédé, marquant l'émergence d'un fond mélancolique, en deçà d'une dépression réactionnelle.

Le transfert aura eu tout au long du traitement un motif principal: une demande paradoxale, visant à me culpabiliser/déculpabiliser: me remercier d'être là pour l'écouter et me reprocher de ne pas être là pour l'entendre. Aura dominé l'ambiance dramatique d'un meurtre à exhiber en le perpétrant sur soi, à défaut d'avoir quelqu'un à tuer qui pourrait y survivre. S'ensuit une troisième année me laissant espérer une sortie au moins partielle du piège narcissique où il s'agissait jusque-là pour la patiente de rechercher quasi exclusivement chez l'analyste ce qui lui faisait défaut comme "personne secourable», au sens de Ferenczi (Ferenczi, 1969, p. 250).

L'ambiance des séances change, le transfert semble réintroduire une présence libidinale minimale. Et j'ai pu trouver alors une certaine satisfaction à me dire, sans doute par anticipation sur mon texte à venir, que la patiente avait été la source vivante d'une focalisation des questions théoriques et cliniques concernant précisément le statut pour les analystes du dialogue fondateur entre Freud et Ferenczi, à la fois créateur et meurtrier. Belle histoire... Peu de temps après, la patiente interrompra brutalement le traitement, sans que rien ne me permette de l'anticiper, en me laissant dans une profonde incertitude quant à sa capacité à se soustraire à la tentation suicidaire.

Aujourd'hui, je dirais que la rupture agie de cette patiente est venue signer la mise en scène d'un voilement/dévoilement chez elle d'une position mélancolique, en forme de revendication. Comme si elle ne pouvait vivre qu'en se voyant intraitable, irréductiblement condamnée à mort dans le regard de l'autre... ce que je n'avais pu voir. Comme si elle était venue de son univers d'enfant incestuée, c'est-à-dire victime d'un meurtre psychique sans témoin, pour confondre en moi, dans tous les sens du terme, l'inanalysé de sa première analyste/mon ex-patiente - pour dénoncer en l'agissant une confusion incestuelle entre l'inanalysé de l'analyste de cette analyste et l'inanalysé de son propre analyste: moi dans les deux cas...

Comment affronter cette forme radicale d'auto-destructivité que Ferenczi le premier a rendu pleinement pensable dans «L'enfant mal accueilli et sa pulsion de mort» (Ferenczi, 1929) ? Une forme d'auto-destructivité bien désignée par l'expression de Schreber, un meurtre d'âme, mais envisagée ici comme un consentement au meurtre psychique, comme un retournement sur soi du meurtre psychique agi originellement par l'autre: une importation 
de la haine meurtrière tuant en soi le besoin de l'autre pour se sentir exister, comme s'il s'agissait de se tuer en l'autre. La haine meurtrière serait ici à envisager comme une modalité de survivance, la tentative de sauver le minimum vital d'investissements libidinaux, dans le choix relationnel d'un autre réduit à réactiver irrémédiablement la défection de l'autre secourable.

Il reste que pour accueillir une telle haine de soi, pour la rendre possible à accueillir par ces patients, pour les accompagner dans le mouvement consistant à la ré-adresser dans le transfert, encore faut-il que l'analyste puisse accueillir sa propre haine, comme le théorise Winnicott dans le texte de 1947. L'enjeu pour l'analyste est de s'accueillir dans ce qui l'affecte de cette scène inaugurale du mal-accueil de l'être, de la haine meurtrière pour soi en tant qu'elle exprime la haine meurtrière de l'autre, de la haine meurtrière pour l'autre en tant qu'elle contient la haine meurtrière de soi. Sans cet accueil, l'adresse transférentielle de la haine ne peut avoir lieu et donc la symbolisation du meurtre ne pourra advenir.

S'accueillir ainsi n'est possible qu'à garder le contact avec l'à-venir du plaisir de penser. Il s'agit d'accueillir son insu transférentiel et la possibilité après coup d'en penser une part, jamais définitivement établie: la possibilité d'en faire une source du penser, énigmatique et instable, mais vivante. Ici, pour moi, l'insu et son après-coup concernait donc le registre mélancolique, sous deux aspects. D’une part, la dimension mélancolique des problématiques limites, c'est-à-dire la dimension de l'absence trouée par le vide, par l'indissociable perte de soi et de l'autre - dimension dont j'ai beaucoup tardé à prendre acte dans ma clinique, quasiment jusqu'à la confrontation clinique ici rapidement évoquée. D’autre part, «le travail de la mélancolie» (Freud, 1917) qui vient s'immiscer dans l'intimité de la position d'analyste, voire, avec les patients limites, la parasiter.

Le travail de la mélancolie ${ }^{1}$ en l'analyste, c'est le détachement de soi et de l'autre, l'activation de l'angoisse de la perte dans l'absence, lorsqu'entre en scène chez le patient le besoin paradoxal de l'effacement de soi en l'autre, qui peut aller jusqu'à la menace de la disparition, dans la forme extrême de l'appel ou de l'injonction à se faire disparaître. Et ce travail de la mélancolie est devenu manifeste pour moi en prenant la mesure, rudement et dans l'après-coup, de la nécessité intérieure qui m'avait amené à prendre le risque de m'adresser à un public à partir de cette clinique-là, en renonçant à la protection que je m’offre généralement avec la médiation des écrivains... Faire de l'absence en soi, là où le transfert du patient limite veut le vide, exigeait ici non seulement le secours de la publication, mais plus précisément 
l'après-coup de l'écriture et sa réitération ${ }^{2}$, dans une modalité trouvant son ancrage dans l'écriture freudienne et non dans la littérature, même sous l'angle bien particulier des écritures de soi.

... C'était donc, pourrait-on dire, une présentation de mon «transfert sur la psychanalyse». Mais il me semble que la relation analytique, impossible et créatrice, de Freud et Ferenczi, dessine pour chaque analyste une scène transférentielle à la fois originelle et originaire - originelle: c'est-à-dire aux débuts de la psychanalyse; originaire: c'est-à-dire réactivée dans et par le fonctionnement même de l'analyste en situation, la réactivation donnant forme à ce qui l'institue comme analyste. Cette scène, dont il faut souhaiter que la matière soit toujours inédite et singulière avec chaque patient, l'analyste en fait l'expérience dans le dialogue intérieur, l'interlocution interne, l'écoute au moins potentielle de son écoute. Ce n'est pas exactement qu'il se parle en séance: les mots qui lui viennent plus ou moins silencieusement ne cessent d'esquisser un faisceau toujours changeant de polarités, lui permettant de contacter ses résistances et ce qui l'anime dans son offre au transfert - offre au transfert qui est aussi une offre de transfert.

L'enjeu est de convoquer un public intérieur, un témoin interne vivant et composite rendant au moins partiellement expérimentable le tiers présent/absent, dans la présence/absence des identifications à ses analystes, de leur possible mise au travail créative. Et avec les fonctionnements limites, c'est sans doute la condition impérative pour transformer le meurtre de la créativité à deux, entre-deux, en meurtre symbolique et symboligène de l'analyste de l'analyste. Le meurtre symbolique et symboligène de l'analyste de l'analyste, soit une visée fantasmatique à l'œuvre chez le patient et l'analyste, mais à déployer sur deux aires que l'analyste a la responsabilité de rendre pleinement différenciables et différenciées, face à l'agir incestuel du patient.

\section{Jean-François Chiantaretto jfchant@wanadoo.fr}

\section{Notes}

1. Qu'il faut pleinement distinguer de la mélancolie, comme l'a définitivement théorisé Benno Rosenberg (Rosenberg, 1991).

2. Pour arriver à cette présentation et son écriture, il m'aura donc fallu en passer par une première présentation et son écriture. 


\section{Références}

Baldacci, J.-L. (2016). L'analyse avec fin. Paris: Presses universitaires de France.

Baldacci, J.-L. (2010). Le CCTP dit «Centre Jean-Favreau». Le Coq-Héron, 201, 61-67.

Chiantaretto, J.-F. (2016). Au commencement était le meurtre. Le Coq-Héron, 224, 10-20.

Chiantaretto, J.-F. (2011). Trouver en soi la force d'exister. Clinique et écriture. Paris: Campagne première.

Chiantaretto, J.-F. (2006). L'écriture du psychanalyste et la séance. Dans J. André et I. Lasvergnas (dir.), La psychanalyse à l'épreuve du malentendu (p. 117-134). Paris: Presses universitaires de France.

Fédida, P. (2002). Le psychanalyste: un état limite? Dans auteur, Transfert et états limites (p. 85-104). Paris: Presses Universitaires de France.

Fédida, P. (1995). Le site de l'étranger. Paris: Presses universitaires de France.

Ferenczi, S. (1969). Journal clinique. Paris: Payot, 1985.

Ferenczi, S. (1929). L'enfant mal accueilli et sa pulsion de mort. Dans auteur (1982), Psychanalyse IV. Paris: Payot.

Freud, S. (1917). Deuil et mélancolie. Dans auteur (1988), Euvres complètes de Freud XIII (p. 259-278). Paris: Presses universitaires de France.

Guyomard, P. (2011). Lacan et le contre-transfert: le contre-coup du transfert. Dans P. Guyomard (dir.), Lacan et le contre-transfert (p. 11-75). Paris: Presses universitaires de France.

Neyraut, M. (1974). Le transfert. Paris: Presses universitaires de France, 1980.

Rosenberg, B. (1991). Masochisme mortifere et masochisme gardien de la vie. Paris: Presses universitaires de France.

Winnicott, D. W. (1967). Le concept de régression clinique opposé à celui d'organisation défensive. Dans auteur (2000), La crainte de l'effondrement et autres situations cliniques. (p. 318-326). Paris: Gallimard.

Winnicott, D. W. (1960). Le contre-transfert. Dans auteur (1969), De la pédiatrie à la psychanalyse (p. 229-236). Paris: Payot.

Winnicott, D. W. (1947). La haine dans le contre-transfert. Dans auteur (1969), De la pédiatrie à la psychanalyse (p. 48-58). Paris: Payot. 